\title{
EFECTOS DEL CONFLICTO ARMADO EN COLOMBIA EN LA COBERTURA EDUCATIVA DEL DEPARTAMENTO DE BOLÍVAR EN EL PERÍODO 1995-2008*
}

\author{
Antonio Rodríguez Sánchez \\ Edwin José Vega Flórez ${ }^{* * *}$ \\ Luis Alvis Estrada ${ }^{* * *}$ \\ Robinson Castro Ávila*****
}

Recibido: octubre 01 de 2010

Aceptado: marzo 22 de 2011

\section{RESUMEN}

El presente estudio estima el impacto del conflicto interno armado en la cobertura en educación en el departamento de Bolívar entre los años 1995 y 2008 a través de un análisis de regresión doble-logarítmico mediante la técnica de Mínimos Cuadrados Ordinarios. Se toman como variables explicativas la tasa de homicidios, de secuestro y de desplazados y una variable dummy la cual representa la política de Seguridad democrática. Mediante el Criterio de Información de Akaike (CIA) se determinó cuál es la mejor regresión. La cobertura educativa reportó una alta correlación con la tasa de secuestrados. El desplazamiento y la seguridad democrática se encontraron asociados a la cobertura educativa. Se observa un impacto positivo de la seguridad democrática, pues en los años en que ésta se empieza a ejecutar la cobertura varía positivamente.

\section{PALABRAS CLAVE}

Economía de la educación, cobertura en educación, conflicto armado, mínimos cuadrados ordinarios.

\section{CLASIFICACIÓN JEL} I29, I21.

\section{CONTENIDO}

Introducción, 1. Diseño metodológico; 2. Cobertura educativa y las variables asociadas a la violencia en Bolívar 1995-2008; 3. Análisis de correlación; 4. Efectos del conflicto armado en la cobertura educativa: estimación econométrica; 5. Conclusiones; Bibliografía; Anexos.

- Artículo de investigación basado en el trabajo de grado titulado Efectos del conflicto armado colombiano en la cobertura educativa del departamento de Bolívar en el período 1995-2008, elaborado entre 2009 y 2010 que fue presentado en la Facultad de Ciencias Económicas de la Universidad de Cartagena como requisito para optar al título de economistas, bajo la asesoría de Robinson Castro Ávila profesor de planta de esta universidad.

* Economista. Universidad de Cartagena, Colombia. Analista de métrica, Observatorio de Cultura Política, Paz, Convivencia y Desarrollo de los Montes de María, Cartagena, Colombia, Dirección postal: Gaviotas Manzana 76, Lote 8 Etapa 7, Cartagena, Colombia. Tel: +57-3005104638. Correo electrónico: rodriguezantonio85@gmail.com.

$* *$ Economista. Universidad de Cartagena, Colombia. Dirección postal: Torices Calle 45 n 13-26 Tel: +57-3016005687. Correo electrónico: edjoveflo@hotmail.com.

*.** Economista. Universidad de Cartagena, Colombia. Magíster Salud Pública, Universidad Nacional, Bogotá, Colombia. Docente Facultades de Enfermería y Medicina, Universidad de Cartagena, Colombia. Miembro del grupo de investigación de Economía de la Salud de la Universidad de Cartagena. Dirección postal: Avenida del Consulado, calle 30 \# 48 -152, Sede Piedra de Bolívar. Universidad de Cartagena Tel: +57-3173009971. Correo electrónico: lalvis20@yahoo.com.

***** Economista, Universidad de Cartagena, Colombia. Especialista en Gestión del Comercio Internacional y Desarrollo Industrial, Universidad de Cartagena, Colombia. Especialista en Estadística Aplicada Universidad del Norte, Barranquilla, Colombia. Docente de la Universidad de Cartagena, Colombia. Director del grupo de investigación Comercio Internacional y Desarrollo Industrial. Dirección postal: Avenida del Consulado, calle 30 \# 48 -152, Sede Piedra de Bolívar, Universidad de Cartagena Tel: +575-6769601. Correo electrónico: castroavila2305@yahoo.es.

Semestre Económico, volumen 14, N²8, pp. 67-76 • ISSN 0120-6346, enero-junio de 2011, Medellín, Colombia 


\section{EFFECTS OF THE COLOMBIAN ARMED CONFLICT IN THE EDUCATIONAL COVERAGE OF THE BOLIVAR DEPARTMENT IN THE PERIOD OF 1995-2008}

\section{ABSTRACT}

The present study estimates the impact of the internal armed conflict in the education coverage in the Bolivar Department between the years 1995 and 2008 through a double logarithmic regression analysis through an ordinary least square method. The explanation variables are; homicides, kidnapping and forced displacement and one dummy variable which is the Democratic Security Policy.) the best regression was determined by the Akaike Information Criteria (CIA). The educational coverage reported a high correlation with the kidnapping rate. Forced displacement and democratic security where associated to educational coverage. A positive impact of the democratic security policy is observed given the fact that since the beginning of its implementation, educational coverage has varied positively.

\section{KEY WORDS}

Education economy, education coverage, armed conflict, ordinary least squares.

\section{JEL CLASSIFICATION}

I29, I2 1

\section{CONTENTS}

Introduction, 1. Methodological design; 2. Education coverage and the variables associated to violence in Bolivar department during 1995-2008; 3. Correlation analysis; 4. Effects of armed conflict in the educational coverage: econometric estimation; 5. Conclusions; Bibliography; Attachment.

\section{EFEITOS DO CONFLITO ARMADO NA COLÔMBIA NA COBERTURA EDUCATIVA DO DEPARTAMENTO DE BOLÍVAR N PERÍODO 1995-2008.}

\section{RESUMO}

O presente estudo estima o impacto do conflito interno armado na cobertura educativa no departamento de Bolívar entre os anos 1995 e 2008 a traves de uma analise de regressão doble-logarítmica mediante a técnica de Mínimos Quadrados Ordinários. Tomam-se como variáveis explicativas a taxa de homicídios, o sequestro e as pessoas deslocadas e uma variável dummy a qual é a politica de Seguridade Democrática. Mediante o critério de informação de Akaike (CIA) determinou-se qual era a melhor regressão. A cobertura educativa reportou uma alta correlação com a taxa de sequestros. A deslocação e a seguridade democrática encontraram-se associadas, dado que naqueles anos que a seguridade democrática foi aplicada, a cobertura variou positivamente.

\section{PALAVRAS-CHAVES}

Economia da educação, cobertura em educação, conflito armado, mínimos quadrados ordinários.

\section{CLASSIFICAÇÃO JEL}

I29, I2 1

\section{CONTEÚDO}

Introdução, 1. Desenho metodológico; 2. Cobertura Educativa e as variáveis associadas à violência em Bolívar 3. Analise de correlação; 4. Efeitos do conflito armado na cobertura educativa: estimação econométrica; 5. Conclusões, Bibliografia, Anexos. 


\section{INTRODUCCIÓN}

Colombia ha experimentado un escenario de conflicto armado desde hace más de 50 años, que continúa vigente, y que sigue afectando de diversas maneras a la sociedad nacional. En la actualidad, se observan diversas manifestaciones de tal conflicto que pueden ser cuantificadas por medio de indicadores (número de homicidios, número de desplazados, número de secuestros, entre otros) que las identifican y permiten desarrollar el análisis.

El conflicto armado en el país se desarrolló inicialmente en zonas rurales, sin embargo, desde mediados de la década de los ochenta ha mostrado una expansión y crecimiento continuos en algunas zonas urbanas. Según CCVICA (2006) los escenarios educativos, incluso, han sido utilizados como resguardos de los grupos armados ilegales, además el país ocupa el cuarto lugar en el mundo en materia de reclutamiento de niños. Franco y otros (2006) indican que es en este proceso cuando la población infantil es privada de las actividades propias de su niñez, entre ellas, la formación educativa. En el departamento de Bolívar la situación no es ajena a la del resto del país, puesto que se presentan continuos enfrentamientos armados de los grupos al margen de la ley.

Teniendo en cuenta lo anterior, el presente artículo analiza la forma en que el conflicto armado ha afectado la cobertura educativa en el departamento de Bolívar. Para desarrollar este propósito se hizo uso de la estadística descriptiva. Por otro lado, se efectuó una estimación econométrica mediante el método de los mínimos cuadrados ordinarios (MCO) con el fin de calcular los parámetros de las variables que afectan la cobertura educativa en el período comprendido entre 1995 y 2008.

Los hallazgos y conclusiones permiten confirmar que las variables del conflicto, que inciden en la cobertura educativa, son las variaciones en la tasa de desplazados y la existencia de la Política de Seguridad Democrática. De la primera se tiene que por cada $10 \%$ de reducción en la tasa de desplazados, la cobertura educativa sería 1,196\% mayor. De la segunda, la seguridad democrática, ha hecho variar positivamente la cobertura en $0,22 \%$, porque genera condiciones propicias de estabilidad y confianza dentro de la población.

El artículo se organiza de la siguiente forma. En la primera sección se presenta El diseño metodológico. Los principales indicadores y antecedentes que permiten describir y enmarcar el problema se exponen en la segunda sección. En la tercera parte del trabajo se realiza un análisis de correlación entre la cobertura educativa y las variables asociadas al conflicto (homicidios, secuestros, desplazamientos y presencia de la Política de Seguridad Democrática) en Bolívar. En la cuarta sección se muestran los resultados del modelo econométrico estimado. Por último, se concluye y se indican las referencias bibliográficas.

En Colombia se han hecho enormes esfuerzos en materia educativa durante las últimas décadas. Estos han estado encaminados principalmente a aumentar la cobertura básica y secundaria en todo el territorio nacional. Se ha presentado un crecimiento sostenido en los alumnos matriculados en primaria a partir de 1990; sin embargo, en los últimos años no se han visto mejoras en las matrículas de este nivel y aún las coberturas son lejanas del 100\%.

En efecto, la cobertura neta en educación entre 1997 y el 2002 en primaria disminuyó al pasar de $83,1 \%$ al $82,4 \%$, mientras que se observa un crecimiento sostenido en el gasto público en educación como porcentaje del PIB, que aumentó de 4,30\% en 1997 hasta 5,46\% en el 2002.

En el departamento de Bolívar, durante la última década, el crecimiento de la violencia ha jugado un rol importante en el aumento de la deserción e inasistencia escolar. Los mecanismos a través de los cuales el conflicto afecta la educación son los problemas de orden público, el desplazamiento forzado, las amenazas y la muerte violenta a familiares, según precisa la CCVICAC (2006). En un año como el 2007, en el departamento de Bolívar se registraron 263 
casos de homicidios, un dato mayor al presentado en el 2008, que fue de 164, lo que muestra una variación negativa cercana al 38\%, tal como afirma ODHC (2009). Para ese mismo año se alcanzó una cifra de 8.617 desplazados, mientras que para el 2008 fueron 4.377. La disminución en las cifras de violencia relacionadas con el conflicto armado se deben al incremento del pie de fuerza militar que las estrategias gubernamentales han implantado en la zona sur del departamento, caracterizada por ser una de las más violentas de toda la región, según Báez (1999) y ODHC (2009).

En los municipios del departamento de Bolívar con presencia armada de grupos al margen de la ley, la cantidad de alumnos matriculados creció un 1,11\% al año, dice CCVJCAC (2006), mientras que los municipios sin actividad armada lograron alcanzar mayores niveles de asistencia escolar, al crecer a una tasa promedio de 2,58\%. Por lo tanto, se perdieron 1,47 puntos porcentuales de crecimiento, como resultado del entorno desfavorable generado por la actividad de las FARC.

Según González (2006, p. 9) el crecimiento de algunos países se afecta tanto de forma directa como indirecta por parte de algunos hechos violentos, tales como actividad armada, y se han encontrado diferentes conductos que explican de alguna manera este fenómeno: "destrucción de infraestructura, destrucción de capital social, inestabilidad política, desviación de recursos hacia actividades menos productivas y disminución en la productividad de los factores".

El papel de la inversión en capital físico y social es prioritario para la consolidación de los procesos educativos integrales en zonas vulneradas por la violencia. De acuerdo con González (2006), la destrucción de capital social, así como la incapacidad institucional para recuperarlo, generan muertes, emigración de ciudadanos, deserción escolar y desplazamiento forzado. Por otro lado, la inestabilidad política se torna en una barrera para la inversión tanto en educación, como en salud e infraestructura.
Diversos autores han estudiado el impacto del conflicto armado en las sociedades en general y en la educación en particular. Trabajos como el de Shemyakina (2006) reportan los daños en los hogares de Tayikistán durante los conflictos civiles al mostrar un efecto negativo importante en la matrícula de las niñas de edades 12-15 años, y poco, o nulo efecto sobre la matrícula de niños y niñas más jóvenes.

Sommers (2002) considera que no se puede obtener cobertura universal en educación en los países en conflicto puesto que son pocas las guerras en las cuales no se involucra la población escolar.

En Colombia, en el 2004, existían cerca de quinientos mil niños en edad escolar que no estaban incluidos en el sistema educativo, y las autoridades señalan que uno de los determinantes del descenso de la cobertura educativa son los efectos disruptivos del conflicto armado (los otros son la pobre calidad de la educación y las malas condiciones económicas) WCAC (2004) y UNSC(2009). Del mismo modo, Álvarez y Rettberg (2008) analizaron a la comunidad académica y profesional considerando los costos (económicos, políticos y sociales) que enfrentan los diferentes actores combatientes y no combatientes en situaciones de conflicto armado interno para identificar la probabilidad de que ocurra una negociación de paz.

Sánchez y Díaz (2005) señalan que el impacto de la intensidad del conflicto armado colombiano sobre el desarrollo social no ha sido cuantificado rigurosamente y Vargas (2003) dice que el impacto de la intensidad del conflicto armado interno sobre el desempeño económico colombiano no ha sido determinado con precisión en la literatura económica. En este sentido el estudio de Ibáñez y Barrera (2004) desarrolla un modelo teórico dinámico, y presentan evidencia empírica sobre la relación entre la violencia y las inversiones en educación.

En el contexto de los modelos de desarrollo, Benavides y Forero (2002) destacan que uno de los impactos negativos del conflicto armado recae sobre uno de los factores fundamentales para el 
crecimiento económico endógeno como lo es el capital humano.

\section{DISEÑO METODOLÓGICO}

Se trata de un estudio analítico con series de tiempo entre los años 1995-2008. La información utilizada es secundaria y corresponde a datos históricos proporcionados por el Departamento Nacional de Estadística (DANE), Centro de Observación y Seguimiento del Delito (COSED), Secretaría de Educación Departamental, Fondelibertad y la Policía Nacional.

Los datos fueron almacenados y procesados en el programa Microsoft Excel 2007®. Para la estimación del impacto del conflicto armado en la cobertura educativa se empleó un modelo de regresión doble-logarítmico mediante la técnica de Mínimos Cuadrados Ordinarios. La ecuación del modelo está definida como:

\section{In cobertura $_{t}=\alpha_{0}+\alpha_{1} \ln$ homicidio $_{t}$ $+\alpha_{2} \ln$ secuestro $_{t}+\alpha_{3} \ln$ desplazados $_{t}$ $+\alpha_{4}$ seg_democrat}

La variable dependiente es la cobertura educativa (Incobertura), y se encuentra explicada por la tasa de homicidios (Inhomicidio), secuestros (Insecuestro, y desplazados (Indesplazados), todas expresadas en unidades por 100.000 habitantes. Además, se incluye una variable dummy (seg _ democrat) seg _ democrat que toma el valor de 1 para los años en que aparece la política de Seguridad Democrática (2002-2008). Además, con el propósito de hacer más completo el análisis se prueban diversas especificaciones alternativas a la ecuación anterior, y mediante el Criterio de Información de Akaike (CIA) se determina cuál es la mejor especificada. Para el cálculo del CIA se emplea la expresión tomada de Gujarati (2004):

$$
C I A=e^{2 \frac{k}{n}} \frac{\sum \hat{u}_{i}^{2}}{n}
$$

En donde $\mu$ son los términos de error, $k$ es el número de regresores, $n$ corresponde al número de observaciones. Se espera que la relación entre conflicto y cobertura sea inversa, en tanto que ante hechos de violencia, la población en edad escolar desiste de asistir a la escuela.

\section{COBERTURA EDUCATIVA Y LAS VARIABLES ASOCIADAS A LA VIOLENCIA EN BOLÍVAR 1995-2008.}

Al inicio del período de este estudio tanto los homicidios como el desplazamiento y el secuestro experimentan un incremento. En cuanto a la cobertura educativa, se observa un crecimiento entre 1995 y 1998, luego de este año cae y se mantiene más o menos estable entre 1999 y 2001. Una de las posibles causas es la expansión territorial que estaba dándose por parte de los actores armados, así como por la falta de presencia militar en los municipios de la zona.

Las escuelas estaban siendo utilizadas como resguardos e inclusive como sitios de reunión de los actores armados. La presencia de grupos armados en la zona, con sus consecuentes limitaciones impuestas a la libertad, mecanismos coercitivos de control social y constantes hechos violentos, dificultaban la asistencia de los niños a la escuela y generaban en la sociedad síntomas de estrés, depresión e incapacidad para concentrarse en las actividades académicas, según MEN (2009).

Es interesante observar que en los años en que se dan las mayores caídas de las tasas de cobertura bruta en el departamento, también se registra la mayor cantidad de desplazados; esto sucede entre los años 1999 y 2002. Se evidencia que en este mismo período los secuestros y los homicidios muestran valores relativamente altos, tal como se evidencia en el gráfico 1.

Entre los años 1999-2002, la tasa de cobertura bruta promedio resulta ser de $78,17 \%$, inferior a los años anteriores y mucho menor a la del período 2003-2008. Las tasas más altas de homicidios también ocurrieron entre 1999 y 2002. 
Gráfico 1. Evolución de homicidios, desplazados, secuestrados y la cobertura educativa en Bolívar, 1995-2008.

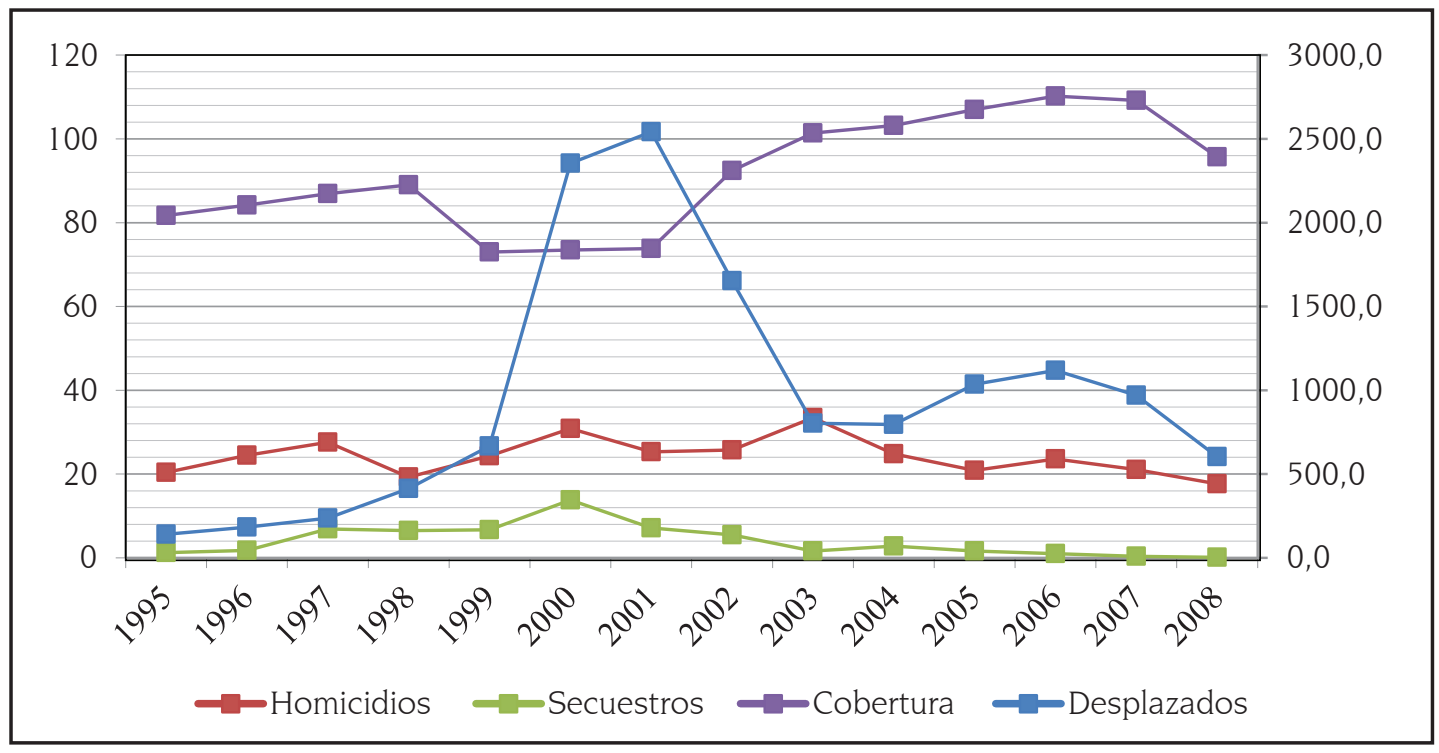

Fuente: cálculos de los autores

\section{ANÁLISIS DE CORRELACIÓN}

La cobertura educativa muestra una mayor correlación con la tasa de secuestrados $(r=-0,69)$. Por su parte, la tasa de homicidios presenta un coeficiente muy bajo $(0,04)$. Todas las correlaciones de la tasas de cobertura con los indicadores del conflicto resultaron con signo negativo, lo que indica que existe una relación inversa entre las variables correspondientes, tal y como se muestra en la tabla 1. Es decir, ante un aumento de cualquiera de los indicadores del conflicto se genera una reducción de la cobertura en educación.

En el mismo cuadro se presentan, además, los coeficientes para el resto de variables. Todas presentan signo positivo; esto se esperaba, pues hechos como los homicidios y el secuestro generan desplazamiento, dado que la gente procura alejarse y proteger su integridad ante hechos violentos, incluso sacrificando lo que más valora. Además, se constata que el grado de la relación es más o menos alto: homicidios y desplazados en 0,43 ; homicidios y secuestrados 0,39; y desplazados 0,54.

\section{EFECTOS DEL CONFLICTO ARMADO EN LA COBER- TURA EDUCATIVA: ESTIMACIÓN ECONOMÉTRICA \\ Antes de mostrar los resultados obtenidos, cabe aclarar que el modelo econométrico que se estimó constituye solo una forma aproximada para conocer}

Tabla 1. Coeficiente de correlación cobertura de educativa y variables del conflicto armado en Bolívar

\begin{tabular}{|l|c|c|c|c|}
\hline \multicolumn{1}{|c|}{ Variables } & $\begin{array}{c}\text { Tasa de cobertura en } \\
\text { educación }\end{array}$ & Tasa de homicidios & Tasa de desplazados & Tasa de secuestrados \\
\hline Tasa de cobertura en educación & 1 & - & - & - \\
\hline Tasa de homicidios & $-0,045703$ & 1 & - & - \\
\hline Tasa de desplazados & $-0,193773$ & 0,431676 & 1 & - \\
\hline Tasa de secuestrados & $-0,693331$ & 0,394701 & 0,5441461 & 1 \\
\hline
\end{tabular}

Fuente: cálculos de los autores 
Tabla 2. Especificaciones al modelo del modelo a estimar

\begin{tabular}{|c|c|}
\hline Especificación & Criterio de información de Akaike \\
\hline $\begin{array}{l}\ln \text { cobertura }_{t}=\mathrm{a}_{0}+\mathrm{a}_{1} \ln \text { homicidio }_{t}+\mathrm{a}_{2} \ln \text { secuestro }_{t}+ \\
\mathrm{a}_{3} \ln \text { desplazados }_{t}+\mathrm{a}_{4} \text { seg_democrat }_{t}+\mathrm{a}_{5} t\end{array}$ & $-31,75$ \\
\hline $\begin{array}{l}\text { lncobertura }{ }_{t}=\alpha_{0}+\alpha_{1} \ln \text { homicidio }_{t}+\alpha_{2} \ln _{\text {secuestro }} \\
+\alpha_{3} \ln \text { desplazados }_{t}+\alpha_{4} \text { seg_democrat }_{t}\end{array}$ & $-30,74$ \\
\hline $\begin{array}{l}\ln \text { cobertura }_{t}=\alpha_{0}+\alpha_{1} \text { homicidio }_{t}+\alpha_{2} \text { secuestro }_{t} \\
+\alpha_{3} \text { desplazados }_{t}+\alpha_{4} \text { seg_democrat }_{t}\end{array}$ & $-30,59$ \\
\hline $\begin{array}{l}\text { cobertura }_{t}=\alpha_{0}+\alpha_{1} \text { homicidio }_{t}+\alpha_{2} \text { secuestro }_{t} \\
+\alpha_{3} \text { desplazados }_{t}+\alpha_{4} \text { seg_democrat }_{t}\end{array}$ & 96,22 \\
\hline $\begin{array}{l}\text { cobertura }_{t}=\alpha_{0}+\alpha_{1} \ln \text { homicidio }_{t}+\alpha_{2} \ln _{\text {secuestro }} t \\
+\alpha_{3} \ln \text { desplazados }_{t}+\alpha_{4} \text { seg_democrat }_{t}\end{array}$ & 99,47 \\
\hline
\end{tabular}

Fuente: cálculos de los autores

la forma en que interactúa con la tasa de cobertura educativa, alguna de las variables asociadas al conflicto interno. Se dice que es un ejercicio aproximado porque los organismos oficiales no tienen una contabilidad precisa de los secuestros, homicidios y desplazados, para un período de tiempo más amplio en Bolívar, y si estos datos existen no se pudo tener acceso a ellos. En este sentido, los resultados del modelo estimado deben interpretarse con precaución; sin embargo, esto no resta poder explicativo a los coeficientes.

Antes de proponer este $\mathrm{u}$ otro modelo como definitivo se probaron especificaciones diferentes, para compararlas entre sí y decidir cuál es la mejor conforme al criterio de información de Akaike, que según Gujarati (2004) es útil para elegir entre modelos rivales. La primera especificación es la mejor dentro de todos los modelos rivales, debido a que presenta el valor más bajo del criterio de información de Akaike $(-31,75)$, por tanto, a la ecuación planteada en la metodología se le añade una variable $t$, que recoge el efecto del tiempo dentro del modelo (ver tabla 2).

El modelo en su conjunto, o de forma global, es significativo (Prob. F < 0,05) y lasvariables se distri- buyen de manera normal (Prob. Jarque Bera $>0,05$ ). Las variables incluidas en la estimación explican la variable dependiente (el logaritmo natural de la tasa de cobertura educativa) en una alta proporción $(\mathrm{R} 2=87,43 \%)^{1}$.

El desplazamiento y la seguridad democrática están asociados a la cobertura educativa. Una variación del $10 \%$ en la tasa de desplazados por cada 100.000 habitantes reduce la tasa de cobertura educativa en 1,196\% en el departamento de Bolívar. De igual forma se observa un impacto positivo del lado de la seguridad democrática, pues en los años en que ésta entra en operación, la cobertura varía positivamente en 0,226\%.

\section{CONCLUSIONES}

Con los resultados del presente estudio se tiene un intento por cuantificar las consecuencias que el conflicto interno colombiano ha tenido sobre la cobertura educativa, específicamente en el departamento de Bolívar. Ésta resulta una variable fundamental para la formación de capital humano, generación de mejor calidad de vida y, a largo plazo, mayor bienestar.

\footnotetext{
Ver los resultados de la estimación del modelo en el anexo.
} 
Tabla 3. Resultados de la estimación econométrica Incobertura

\begin{tabular}{|c|c|c|c|c|c|}
\hline Variable & Coeficiente & $t$ & $p$ & Error estándar & $(p)$ Jarque-Bera \\
\hline Constante & 5,0693 & 6.89 & 0,000 & 0,4421 & - \\
\hline $\ln$ homicidio $_{t}$ & $-0,0365$ & -0.26 & 0,803 & 0,1419 & 0,8829 \\
\hline $\ln$ secuestro $_{t}$ & 0,0509 & 1.45 & 0,179 & 0,0345 & 0,4238 \\
\hline $\ln$ desplazados $_{t}$ & $-0,1196$ & -2.22 & 0,045 & 0,0533 & 0,3114 \\
\hline seg_democrat $t$ & 0,2260 & 2.67 & 0,219 & 0,0182 & 0,6513 \\
\hline$t$ & 0,0243 & 1.38 & & Prob. F $=0,0019$ & \\
\hline $\begin{array}{c}\text { Número de } \\
\text { observaciones }=14\end{array}$ & $\mathrm{R} 2=0,8743$ & $\mathrm{~F}=11,13$ & & & \\
\hline
\end{tabular}

Fuente: cálculos de los autores

En Colombia, las variables del conflicto presentaron cambios significativos que pueden observarse en el período 1997-2003, así: el número de desplazados, creció enormemente entre 1999 (11.839 individuos) y 2000 (42.257 individuos); los homicidios, en el departamento de Bolívar, en 2003, 2000 y 1997, registran los valores máximos de esta variable (617, 554 y 478 personas muertas, respectivamente); en esta época se presenta un recrudecimiento en estas actividades delictivas principalmente por el fortalecimiento, la organización y la expansión de diversos grupos rebeldes. Finalmente, el número de secuestrados ha estado descendiendo a partir del año 2000, muy probablemente como producto de la política de Seguridad Democrática del gobierno del presidente Álvaro Uribe.

También se tiene evidencia de que en promedio, para los años comprendidos entre 1999 y 2002, la tasa de cobertura educativa es menor que en el resto de años, a la vez que los indicadores de violencia resultan presentando los valores más altos.

De los análisis de correlación es posible concluir que los homicidios no muestran una tendencia clara en la forma como se relacionan con la cobertura educativa; el número de secuestrados y el número de desplazados se relacionan negativamente. En especial se recomienda que en futuras investigaciones se estudie este comportamiento, que a pesar de las hipótesis planteadas en el documento, no se tiene certeza respecto a esta dinámica.

Finalmente, se confirma que las variables del conflicto, que inciden en la cobertura educativa, son las variaciones en la tasa de desplazados (por 100.000 habitantes) y la existencia de la Política de Seguridad Democrática. De la primera se tiene que por cada $10 \%$ de reducción en la tasa de desplazados la cobertura educativa sería 1,196\% mayor. La seguridad democrática ha hecho variar positivamente la cobertura en $0,22 \%$, porque genera condiciones propicias de estabilidad y confianza dentro de la población, así la asistencia a la escuela se incrementa.

\section{BIBLIOGRAFÍA}

Álvarez, S. y Rettberg, A. (2008). Cuantificando los efectos económicos del conflicto. En: Colombia Internacional, No 67, pp. 14-37.

Báez, J. (1999). Evolución de indicadores educativos para Bolívar y Cartagena (1933-1995) y determinantes regionales. Serie de Estudios Sobre la Costa Caribe. Cartagena de Indias: Universidad Jorge Tadeo Lozano, Seccional del Caribe. Departamento de Investigaciones.

Benavides, O. y Forero, C. (2002). Crecimiento endógeno: Conocimiento y patentes. En: Revista de Economía Institucional, Vol. 4 No 6, pp. 109-131. 
CCVICA -Coalición Contra la Vinculación de Jóvenes al Conflicto Armado en Colombia- (2006). Informe sobre la situación de derechos humanos de los jóvenes de la región de la Costa Caribe, Bogotá D. C., 92p.

Franco, S., Suárez, C., Naranjo, C., Báez, L. y Rozo, P. (2006). The effects of the armed conflict on the life and health in Colombia. En: Ciencia e Saúde Coletiva, Vol. 11 No 2, p.349-361.

González, Á. (2006). Convergencia regional y conflicto armado interno en Colombia, 1980-2004. Facultad de Ciencias Economicas y Administrativas. Bogotá D. C.: Pontificia Universidad Javeriana, 31p.

Gujarati, D. (2004). Econometría,4a ed., México: McGrawHill, 972p.

Ibáñez, A. y Barrera, F. (2004). Does violence reduce investment in education?: a theoretical and empirical approach. Documentos CEDE, Bogotá D. C.: Universidad de los Andes, 24p.

MEN -Ministerio de Educación Nacional- (2009). No más niños en el conflicto. Bogotá D. C., 105p.

ODHC -Observatorio de los Derechos Humanos en Colombia- (2009). Indicadores sobre derechos humanos y DiH Colombia. Bogotá D. C.: Observatorio del
Programa Presidencial de derechos humanos y DIH, 62p.

Sánchez, F. y Díaz, A. (2005). Los efectos del conflicto armado en el desarrollo social colombiano, 1990-2002. Investigaciones y publicaciones CEDE Bogotá D. C.: Universidad de los Andes, 24p.

Shemyakina, O. (2006). The effect of armed conflict on accumulation of schooling: results from Tajikistan. $\mathrm{HiCN}$ Working Papers, Households in Conflict Network, 63p.

Sommers, M. (2002). Children, education and war: reaching education for all (EFA) objectives in countries affected by conflict. Working Papers, Conflict Prevention and Reconstruction Unit, 48p.

UNSC -United Nations Security Council- (2009). Report of the secretary: general on children and armed conflict in Colombia. United Nations Security Council, 2p.

Vargas, J. (2003). Conflicto interno y crecimiento económico en Colombia. Bogotá D. C.: Universidad de los Andes, 85p.

WCAC -Watchlist on Children and Armed Conflict(2004). Colombia's war on children. New York, Watchlist on Children and Armed Conflict, 47p. 
ANEXOS: resultados de las regresiones

\begin{tabular}{|c|c|c|c|c|c|c|}
\hline Source & $8 S$ & $d f$ & \multirow{2}{*}{$\frac{15}{.04970033}$} & & \multirow{3}{*}{\multicolumn{2}{|c|}{ 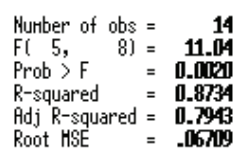 }} \\
\hline $\begin{array}{r}\text { Hodel } \\
\text { Residual }\end{array}$ & $\begin{array}{l}.2485041486 \\
.036006361\end{array}$ & $\begin{array}{ll}5 & .06 \\
8 & 0\end{array}$ & & & & \\
\hline Total & .2845115508 & 13. & 85424 & & & \\
\hline ln_cobertura & Coef. & Std. Err. & $t$ & $p>i t i$ & [95\% Conf. I & Interval ] \\
\hline $\begin{array}{r}\text { ln_horicid"s } \\
\text { lndesplaz"s } \\
\text { ln_secuest"s } \\
\text { seg_democ } \\
t \\
\text { _cons }\end{array}$ & 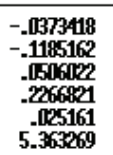 & 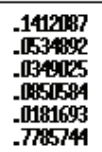 & $\begin{array}{l}-1.26 \\
-2.22 \\
1.45 \\
2.6 \\
1.37 \\
6.89\end{array}$ & $\begin{array}{l}0.798 \\
0.058 \\
0.185 \\
0.029 \\
0.204 \\
0.000\end{array}$ & 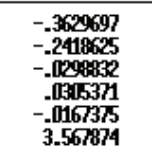 & 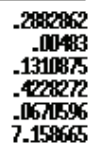 \\
\hline
\end{tabular}

. estat ic

\begin{tabular}{r|rrrrrr}
\hline Hodel & 0bs & 11 (null) & 11 (nodel) & $\mathrm{df}$ & AIC & BIC \\
\hline. & $\mathbf{1 4}$ & $\mathbf{7 . 4 7 4 5 7}$ & $\mathbf{2 1 . 8 7 6 6 8}$ & $\mathbf{6}$ & $\mathbf{- 3 1 . 5 3 3 6}$ & $\mathbf{- 2 7 . 9 1 9 0 2}$ \\
\hline
\end{tabular}

\begin{tabular}{|c|c|c|c|c|c|c|}
\hline Source & SS & $d f$ & HS & & \multirow{3}{*}{$\begin{array}{l}\text { Murber of obs }= \\
\mathrm{Fl} 4, \quad 9 \mathrm{~g}= \\
\text { Prob }>\mathrm{F} \\
\mathrm{R}-\text {-squared } \\
\text { Adj R-squared } \\
\text { Root HSE }\end{array}$} & \multirow{3}{*}{$\begin{aligned}= & 14 \\
= & 11.17 \\
= & 0.0015 \\
= & 0.8324 \\
= & 6.5692\end{aligned}$} \\
\hline $\begin{array}{r}\text { Hodel } \\
\text { Residual }\end{array}$ & $\begin{array}{l}1925.18234 \\
387.67496\end{array}$ & \multicolumn{2}{|c|}{$\begin{array}{lr}4 & 401.205586 \\
9 & 43.074944\end{array}$} & & & \\
\hline Total & 2312.85694 & $\begin{array}{ll}13 & 177\end{array}$ & 12065 & & & \\
\hline cobertura & Coef. & Std. Err. & $t$ & $P>i t i$ & [95\% Conf. I & nterval ] \\
\hline $\begin{array}{r}\text { homicidios } \\
\text { desplazados } \\
\text { secuestrados } \\
\text { seg_denoc } \\
\text { _cons }\end{array}$ & $\begin{array}{l}-.0101695 \\
-.0010955 \\
-.0183832 \\
21.43468 \\
86.53154\end{array}$ & $\begin{array}{l}.0280968 \\
.0001948 \\
-10519108 \\
5.6133 \\
10.61285\end{array}$ & $\begin{array}{r}-0.08 \\
-1.00 \\
-0.00 \\
3.75 \\
8.16\end{array}$ & $\begin{array}{l}0.941 \\
0.942 \\
0.731 \\
0.0404 \\
0.000\end{array}$ & $\begin{array}{r}-.0657289 \\
-.0016361 \\
-.1358136 \\
\mathbf{8 . 6 1 5 4 4 5} \\
62.54361\end{array}$ & $\begin{array}{l}.0613898 \\
.0002452 \\
.0909473 \\
34.26432 \\
110.5595\end{array}$ \\
\hline
\end{tabular}

. estat ic

\begin{tabular}{r|rrrrrr}
\hline Hodel & Obs & 11 (nul1) & ll(nodel) & df & AIC & BIC \\
\hline$\cdot$ & $\mathbf{1 4}$ & $\mathbf{5 5 . 6 1 5 4 1}$ & $\mathbf{- 4 3 . 1 1 2 9}$ & $\mathbf{5}$ & $\mathbf{9 6 . 2 2 5 8}$ & $\mathbf{9 9 . 4 2 1 0 9}$ \\
\hline
\end{tabular}

- regress ln_cobertura ln_horicidios ln_desplazados ln_secuestrados seg_denoc

\begin{tabular}{|c|c|c|c|c|}
\hline Source & SS & $d f$ & HS & Hunber of obs $=14$ \\
\hline $\begin{array}{r}\text { Hodel } \\
\text { Residual }\end{array}$ & $\begin{array}{l}.239873904 \\
.044635504\end{array}$ & $\begin{array}{l}4 \\
9\end{array}$ & . 159968251 & $\begin{array}{l}\text { Prob }>F=0.0012 \\
\text { R-squared }\end{array}$ \\
\hline Total & .2845115080 & 13 & . 102885424 & Root HSE \\
\hline
\end{tabular}

\begin{tabular}{|c|c|c|c|c|c|c|}
\hline In_cobertura & Coef. & Std. Err. & $t$ & P>iti & [95\% Conf & Interval] \\
\hline $\begin{array}{l}\text { ln_horicid"s } \\
\text { ln_desplaz"s } \\
\text { ln_secuest"s } \\
\text { seg_deroc }\end{array}$ & 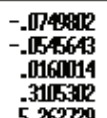 & $\begin{array}{l}.1554615 \\
.0283314 \\
.0255821 \\
.06627113 \\
.9127430\end{array}$ & $\begin{array}{r}-0.52 \\
-1.93 \\
0.63 \\
4.95 \\
6.9\end{array}$ & $\begin{array}{l}0.619 \\
0.086 \\
0.547 \\
0.001 \\
0.001\end{array}$ & $\begin{array}{r}-.4041937 \\
-.1186543 \\
-.0418693 \\
.1696674 \\
-3.01012\end{array}$ & $\begin{array}{l}.254075 \\
.0095258 \\
.0738721 \\
.452393 \\
.10254\end{array}$ \\
\hline
\end{tabular}

. estat ic

\begin{tabular}{r|rrrrrr}
\hline Hodel & Obs & 11 (nul1) & 11 (nodel) & df & AIC & BIC \\
\hline. & $\mathbf{1 4}$ & $\mathbf{7 . 4 7 4 5}$ & $\mathbf{2 0 . 3 7 2 5 3}$ & $\mathbf{5}$ & $\mathbf{- 3 0 . 7 4 5 0 6}$ & $\mathbf{- 2 7 . 5 4 9 7}$ \\
\hline
\end{tabular}

- regress cobertura ln_horicidios ln_desplazados lr_secuestrados seg_democ

\begin{tabular}{|c|c|c|c|c|c|c|}
\hline \multirow{3}{*}{$\begin{array}{r}\text { Source } \\
\begin{array}{r}\text { Hodel } \\
\text { Residual }\end{array} \\
\text { Total }\end{array}$} & SS & \multirow{2}{*}{\multicolumn{2}{|c|}{$\begin{array}{cc}d t & \\
4 & 479.545865 \\
9 & 43.8525979\end{array}$}} & & \multirow{3}{*}{$\begin{array}{l}\text { Munber of obs } \\
F(4, \quad 9) \\
\text { Prob }>F \\
R \text { R-squared } \\
\text { Adj R R-squared } \\
\text { Root HSE }\end{array}$} & \multirow{3}{*}{$\begin{array}{l}=14 \\
=\quad 10.94 \\
=0.0017 \\
=0.8294 \\
=0.7535 \\
=6.6221\end{array}$} \\
\hline & $\begin{array}{l}\text { 1918.18346 } \\
\text { 394.673981 }\end{array}$ & & & & & \\
\hline & 2312.85694 & 1317. & 12165 & & & \\
\hline cobertura & Coef. & Std. Err. & $t$ & $P>i t i$ & [95\% Conf. & Interval] \\
\hline $\begin{array}{l}\text { ln_horicid"s } \\
\text { ln_desplaz"s } \\
\text { ln_secuest "s } \\
\text { seg_denoc } \\
\text { _cons }\end{array}$ & $\begin{array}{c}-6.2011527 \\
-4.220563 \\
1.212249 \\
22.2716 \\
151.0441\end{array}$ & $\begin{array}{l}13.6782 \\
2.6641818 \\
2.415498 \\
5.8967 / 7 \\
6.516 \% 8\end{array}$ & $\begin{array}{r}-0.45 \\
-1.59 \\
0.50 \\
4.62 \\
1.97\end{array}$ & $\begin{array}{l}0.661 \\
0.147 \\
0.629 \\
0.001 \\
0.080\end{array}$ & $\begin{array}{r}-37.14201 \\
-10.25399 \\
-4.2393666 \\
13.93216 \\
-22.048986\end{array}$ & $\begin{array}{l}24.73986 \\
1.796864 \\
6.643865 \\
11.61104 \\
324.1371\end{array}$ \\
\hline
\end{tabular}

. estat ic

\begin{tabular}{r|rrrrrr}
\hline Hodel & 0bs & 11 (null) & 11 (nodel) & df & AIC & BIC \\
\hline. & $\mathbf{1 4}$ & -35.61541 & $-\mathbf{4 3 . 2 3 9 1 5}$ & $\mathbf{5}$ & $\mathbf{9 6 . 4 6 3}$ & $\mathbf{9 9 . 6 7 5 8}$ \\
\hline
\end{tabular}

\begin{tabular}{|c|c|c|c|c|c|c|}
\hline Source & ss & df & HS & & \multirow{3}{*}{$\begin{array}{l}\text { Hunber of obs = } \\
\text { Fi 4, 9] } \\
\text { Prob }>\mathrm{F}= \\
\text { R-squared } \\
\text { Adj R-squared } \\
\text { Root HSE }\end{array}$} & \multirow{3}{*}{$=\begin{array}{r}11.9 \\
0.0019 \\
0.8414 \\
0.7199 \\
0.0108\end{array}$} \\
\hline $\begin{array}{r}\text { Hodel } \\
\text { Residual }\end{array}$ & $\begin{array}{l}.239398891 \\
.055117707\end{array}$ & 9 & . 159984822 & & & \\
\hline Total & .284511508 & 13 & .1018285424 & & & \\
\hline In_cobertura & Coef. & Std. Err. & t & $P>i t i$ & [95\% Conf. I & Interval ] \\
\hline $\begin{array}{r}\text { horicidios } \\
\text { desplazados } \\
\text { secuestrados } \\
\text { seq_democ } \\
\text { _cons }\end{array}$ & $\begin{array}{l}-.0000319 \\
-2063-166 \\
-.0001586 \\
.2411024 \\
4.45807\end{array}$ & $\begin{array}{l}.0003031 \\
2.11 \mathrm{Re}-166 \\
.00156 \\
.0611822 \\
.114912\end{array}$ & $\begin{array}{r}-0.11 \\
-1.25 \\
-0.28 \\
3.94 \\
38.94\end{array}$ & $\begin{array}{l}0.919 \\
0.242 \\
0.783 \\
0.003 \\
0.000\end{array}$ & 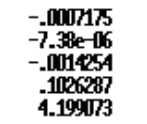 & $\begin{array}{r}.0006538 \\
2.12 \mathrm{e}-16 \\
.0011083 \\
.379436 \\
4.717067\end{array}$ \\
\hline
\end{tabular}

. estat ic

\begin{tabular}{r|rrrrrr}
\hline Hodel & Obs & 11 (nul1) & 11(nodel) & df & AIC & BIC \\
\hline$\cdot$ & $\mathbf{1 4}$ & $\mathbf{7 . 4 7 4 5}$ & $\mathbf{2 1 . 2 9 7 6 3}$ & $\mathbf{5}$ & $\mathbf{- 3 0 . 5 9 5 2 5}$ & $\mathbf{- 2 7 . 3 9 9 9 6}$ \\
\hline
\end{tabular}

